\title{
RUTAS DE ACCESO SEMÁNTICO DE LA ESCRITURA «kanji» EN LECTORES NO NATIVOS: ¿VÍA FONOLÓGICA O VÍA ORTOGRÁFICA? CREENCIAS Y ESTRATEGIAS
}

\section{Edinson Muñoz Arias**}

\section{Resumen}

Este artículo presenta los resultados parciales de una investigación que tiene como objetivo estudiar el papel que juega la ortografía como activador semántico en la comprensión lectora de textos en japonés por parte de lectores no nativos. En esta primera parte, se intenta establecer la relación que tienen las creencias de los lectores sobre cómo funciona el sistema de escritura logográfico y las estrategias para acceder al significado que utilizan con mayor frecuencia. Los resultados sugieren que los lectores principiantes de japonés tienden a acceder al significado por la vía fonológica y visual simultáneamente, a pesar de su firme convicción de que al leer kanji los decodifican directamente como una información semántica.

Palabras clave: kanji, escritura logográfica, ortografía.

\section{WAYS OF SEMANTIC ACCESS OF «kanji» WRITING IN NON- NATIVE SPEAKERS: PHONOLOGICAL OR ORTHOGRAPHIC? BELIEFS AND STRATEGIES}

\begin{abstract}
This article presents the partial results of a study that aims to understand the role orthography as a semantic activator in Japanese texts as part of the reading comprehension process in Chilean Japanese language learners. This first part of the study aims at stablishing a link between the students' beliefs as to how the logographic system works and the strategies they frequently use to access their meaning. The results suggest that beginners tend to associate meaning by using phonologic and visually means simultaneously in spite of their conviction that, as they read, they process kanji characters as a logographic image and decode semantic information directly.
\end{abstract}

Keywords: kanji, logographic system, orthography.

Recibido: 22-01-2016

Aceptado: 16-06-2016

Este artículo se inscribe en el proyecto de investigación Rutas de acceso semántico de la escritura morfográfica del japonés 'Kanji' como segunda lengua: ¿vía fonológica o vía ortográfica? Proyecto DICYT N ${ }^{\circ}$ 031551MA, Vicerrectoría de Investigación, Desarrollo e Innovación de la Universidad de Santiago de Chile.

* Chileno. Magíster en Lingüística. Académico del Departamento de Lingüística y Literatura de la Universidad de Santiago de Chile, Santiago, Chile. edinson.munoz@usach.cl 


\section{Introducción}

La idea de que existen dos vías para la identificación de las palabras se origina en los años 70 cuando se introduce el modelo de procesamiento dual, que postula que la identificación de las palabras para acceder al significado envuelve dos estrategias: la fonológica y la visual (Baron, 1973; Coltheart, 1978). Estudios posteriores revelaron que el grado en que cada una de estas estrategias estaba envuelta en la lectura variaba de acuerdo a las características ortográficas de las lenguas. Por ejemplo, la Hipótesis de la Profundidad Ortográfica propuso que existían tanto una ortografía profunda y otra superficial. La diferencia radicaría en que existen ortografías que activan la información fonológica antes del acceso léxico, mientras que otras lo hacen después (Durgunoglu y Oney, 1999; Frost, 1994). De acuerdo a esta teoría, la ortografía utilizada en las lenguas alfabéticas (ortografía superficial), como la del español, envuelve un tipo de fonología preléxica, por cuanto existe una mayor relación entre el grafema y el fonema que hace que la ruta para llegar al significado de la palabra sea ante todo fonológica, es decir, está basada en el sonido, prescindiendo que la lectura sea en silencio. Por otra parte, un lenguaje que utiliza un sistema de escritura no alfabético, en este caso logográfico o morfográfico, como el chino y el japonés, implicaría un sistema de fonología postléxica, es decir, el proceso cognitivo envuelto en su lectura implicaría decodificar el significado a través de la representación visual de la letra escrita sin mediación fonológica necesariamente. En este caso, la palabra es una unidad completa que se relaciona directamente con el significado.

De la misma forma, Valaki y otros (2003) han clasificado las lenguas de acuerdo a los sistemas de escritura con los que están asociados. Sobre la base de ese criterio las han clasificado de la siguiente forma: (1) sistema alfabético con lectura parcialmente morfográfica, como el inglés; (2) sistema alfabético con reglas rígidas de correspondencia entre grafema y fonema, como el español y el italiano; (3) sistema silábico y logográfico mixto, como el japonés; y (4) sistema logográfico, como el chino. La clasificación responde a los distintos perfiles de activación cerebral descritos de acuerdo a las pruebas magnetoenfalográficas aplicadas a sujetos leyendo diferentes sistemas de escritura. Ahora bien, esto es lo que se ha investigado con respecto a la identificación de las palabras en 
lengua materna. Pero, ¿Qué sucede cuando el lector lo hace como una segunda lengua?

Algunas investigaciones, como las de Chikamatsu (1996) y Koda (1996, 1999) que han estudiado lenguas con sistema de escritura logográfico como el chino, han sostenido la idea de que las estrategias que los sujetos utilizan en la lectura en su lengua materna influyen en la lectura de la segunda lengua. Esto quiere decir que, si un lector con antecedentes alfabético o fonográfico, como el español, aprende chino o japonés utilizará estrategias fonológicas para el reconocimiento de las palabras.

No obstante, estudios más recientes han presentado resultados diferentes. Akamatsu (2002) examinó los procedimientos de identificación de palabras que seguían sujetos con diferentes sistemas de escritura y concluyó que las diferencias ortográficas de la lengua materna no influían en la identificación de palabras de la segunda lengua. Según explica, esto podría deberse a la suficiencia de los sujetos en la segunda lengua o bien, a que los mecanismos de procesamiento de las palabras son universales.

Otro antecedente pertinente fue el de Chikamatsu (2006), quien no sólo investigó el efecto del reconocimiento de palabras de la lengua materna en la segunda lengua, sino que además, comparó la situación con sujetos que estaban en diferentes etapas de aprendizaje. Los resultados mostraron que el nivel de dominio y el tiempo dedicado al estudio del japonés influían en la tarea de identificación de palabras durante la lectura. Los sujetos con mayor suficiencia dependían preferentemente de claves visuales para desempeñar bien la tarea. A partir de estas evidencias, su conclusión fue que los sujetos con el tiempo reconstruyen sus estrategias de reconocimiento de palabras en la segunda lengua y esto les permite amortiguar los efectos de la lengua materna. De la misma forma, Koda (2000) ha sostenido que la experiencia en procesar textos en la segunda lengua es la que permite finalmente evitar la influencia de la lengua materna.

En vista de estos antecedentes, este estudio aspira a esclarecer qué sucede con los estudiantes de japonés como segunda lengua que han tenido la instancia de aprender el sistema de escritura logográfico japonés de forma simultánea a aprender la lengua hablada (esto pudiera 
parecer lógico, pero no lo es, puesto que en algunos lugares se privilegia la adquisición de la lengua, y de forma posterior la de la grafémica). Además, se han estimado diferentes variables para determinar qué estrategias utilizan los lectores de japonés como segunda lengua. En primer lugar, el nivel de suficiencia de los estudiantes. ¿Utilizan las mismas estrategias de reconocimiento de las palabras los lectores de niveles elementales y los más avanzados? ¿Operan de la misma forma la vía fonológica y la semántica en estos casos? ¿Qué sucede con las creencias que tiene el aprendiz de un sistema logográfico a la hora de leer un texto en japonés? Si considera el sistema morfográfico como básicamente un sistema visual-semántico, donde los caracteres los ve como símbolos que transmiten el significado prescindiendo de su fonología, ¿incidirá este hecho en leer con comprensión en silencio, pero no ser capaz de reproducirlo fonéticamente? En segundo lugar, otra variable a considerar, es el objetivo con que los sujetos están aprendiendo japonés como segunda lengua. Existen muchos casos en que las personas necesitan leer en una segunda lengua, como sucede en caso de personas que requieren leer inglés para comprender manuales $u$ otro tipo de escritos, pero no necesariamente desarrollan un nivel de suficiencia en esa lengua que les permita comunicarse oralmente. A la vez, en el caso del japonés se da con frecuencia el caso de personas que hablan fluidamente el idioma, pero no son capaces de leer un texto escrito en kanji (escritura logográfica). ¿Podría darse el caso inverso? Tomando en consideración las características de un sistema morfográfico como el kanji, ¿Podría darse el caso que un principiante o incluso un lector más avanzado desarrollara las habilidades y estrategias para leer comprensivamente un texto en japonés, pero no pudiera reproducirlo acústicamente? Las respuestas a estas preguntas podrían entregar claves para determinar hasta qué grado los lectores pueden prescindir de la fonología en un sistema morfográfico.

Este estudio de caso se ha enfocado en responder parcialmente estas preguntas, pero integrando cada uno de estos aspectos. En primer lugar, los participantes responden a un perfil determinado: son estudiantes universitarios de japonés como segunda lengua de nivel básico que se están iniciando en un programa de traducción. Es decir, a pesar de que las habilidades comunicacionales en la segunda lengua son elementales, la habilidad de comprensión lectora juega un papel preponderante en 
su formación como futuro traductores. En segundo lugar, su proceso de adquisición de la lengua se ha desarrollado en un país donde la práctica misma del idioma es bastante limitada. $Y$ en tercer lugar, todos tienen una concepción bastante definida de cómo funciona para ellos el sistema de escritura logográfico.

Este último aspecto recibe una consideración especial en el presente informe. En el último tiempo los estudios sobre el papel que juegan las creencias de los estudiantes sobre la naturaleza del aprendizaje de una lengua y la eficacia de ciertas estrategias a la hora de adquirir habilidades lingüísticas han constatado que estas tienen un efecto significativo en el conocimiento del vocabulario (Gu y Johnson, 1996) y la comprensión lectora (Carrell, 1989), entre otras habilidades. Mori (1999) en un estudio donde investigó cómo se relacionan las creencias sobre el aprendizaje de la lengua y su relación con la habilidad de integrar vocabulario a partir de los morfemas, llegó a la conclusión de que las estrategias de inferencia de las palabras por parte de los estudiantes de japonés reflejan en algún grado sus creencias sobre el aprendizaje de la lengua. Sobre la base de estos hallazgos, este informe preliminar intenta establecer la relación que tienen las creencias de los estudiantes sobre cómo funciona el sistema de escritura logográfica kanji y las estrategias para acceder al significado que utilizan con frecuencia.

Este estudio surge de la intención de esclarecer el escenario de los estudiantes universitarios chilenos de japonés, pues en su proceso de aprendizaje del sistema logográfico aparecen de forma reiterada situaciones que hacen pensar que probablemente el acceso semántico es mucho más directo y que no dependen de la vía fonológica al leer kanji. En primer lugar, está el concepto mismo que los alumnos tienen acerca del sistema de escritura kanji. La mayoría de ellos, si no todos, abrigan la idea de que kanji es un tipo de escritura ideográfica con la que pueden interpretar el significado casi de forma automática. Este concepto se ve en muchas ocasiones respaldado por el hecho de que cuando van a leer no recuerdan la lectura de cierta palabra, pero sí recuerdan su significado. Incluso en las instancias de evaluación sumativa, las respuestas correctas casi siempre son más aquellas que tienen que ver con recordar el significado más que con recordar cómo se leía determinada letra o palabra. En segundo lugar, otra situación interesante que se 
presenta no pocas veces, es que cuando se les pide leer una oración o texto olvidan la lectura de algunas letras, pero son capaces de recordar su significado. De ahí que la mayoría prefiera leer en silencio, más bien que reproducir acústicamente un texto. De hecho, es común escuchar entre los estudiantes que cuando leen en voz alta un texto se les hace más difícil comprender su significado, pues mientras leen ponen toda su atención en recordar las lecturas, lo que provoca que desplacen a un segundo plano la lectura comprensiva.

Con el fin de ir esclareciendo las estrategias de reconocimiento de palabras de los estudiantes chilenos de japonés al momento de leer $k a n j i$, este estudio de caso se enfoca en entregar algunas luces sobre las estrategias que utilizan con frecuencia dichos lectores del sistema morfográfico japonés como segunda lengua y compararlas con las creencias que ellos tienen del mismo.

\section{Antecedentes}

En esta sección se presenta un marco referencial que aborda dos aspectos: por una parte, una discusión sobre la trascendencia de la vía fonológica en la lectura de un sistema de escritura logográfico o morfográfico, y luego, se presentan antecedentes adicionales a los de la sección anterior sobre las estrategias que siguen los lectores de japonés o chino como segunda lengua con respecto a la identificación de las palabras.

Los sistemas de escritura que existen en el mundo podrían dividirse en tres tipos: el alfabético, el silábico y el logográfico o morfográfico (DeFrancis, 1989). En el primero, la relación entre el grafema y el fonema es bastante estrecha, como sucede con el sistema de escritura del español. En el segundo tipo, un ejemplo de sistema de escritura silábico es el hiragana y katakana utilizados en el idioma japonés, donde cada grafema corresponde a una sílaba del lenguaje hablado. Y por último, un sistema logográfico, en el cual cada grafema representa un morfema o unidad con significado. El kanji japonés o handzi chino es un ejemplo de sistema de escritura logográfico. A pesar de las características de este último, en los tres existe una relación fonológica-ortográfica (Matsunaga, 1996). De hecho, se ha postulado el «principio fonológico universal» (Perfetti y otros, 1992), el que establece que en cualquiera de los tres sistemas 
de escritura, las propiedades fonológicas se activan automáticamente al momento de decodificar la palabra escrita. En el caso de los sistemas alfabéticos y silábicos no quepa ninguna duda de que es así, pero en el caso de un sistema logográfico, ¿existe realmente consenso en que la vía fonológica es obligatoria para acceder al significado de la palabra?

En la actualidad, la tesis de activación de la vía fonológica en la lectura de kanji goza de amplia aceptación. La premisa de varios estudios ha sido «leer chino es similar a leer inglés», tal como lo afirmara Richard Vanness (2005) en su comentario sobre el libro de Mary Erbaugh, publicación que trata de diferentes estudios sobre el sistema de escritura chino y japonés (2002). De acuerdo a los diferentes ensayos que presenta este libro, la idea de que el japonés y el chino presentan un sistema de escritura que permite acceder a los significados sin seguir la ruta fonológica es un «mito» fuertemente arraigado entre lingüistas sensacionalista del pasado que vieron el sistema de escritura chino y japonés como algo místico. Con el propósito de desmitificar esta idea, los autores exploran desde perspectivas sociolingüísticas y psicolingüísticas las razones de por qué este mito ha subsistido por tanto tiempo (Unger, 1990). No obstante, los intentos por explicar el mito no han quedado sólo en eso.

Los estudios sobre los procesos cognitivos envueltos en la lectura del japonés y del chino han tratado de establecer como un hecho que los lectores siguen la vía indirecta del procesamiento semántico, es decir, que al momento de leer los caracteres kanji se activa de forma automática la vía fonológica para así acceder al significado. En la misma línea, Kess y Miyamoto (1999) sostienen que el sistema de escritura japonés está supeditado a un tipo de procesamiento universal, pues el acceso al lexicón mental ocurre de forma directa e indirecta en cualquier sistema de escritura, no sólo en el sistema logográfico. De la misma forma, Widell y otros (1993) y Morita y Tamaoka (2002) concluyen que tanto el proceso fonológico como ortográfico posibilitan el acceso léxico en el caso de la escritura logográfica del japonés. De hecho, se piensa que este proceso tiene su correlato en las lenguas con un sistema de escritura alfabético. Se ha postulado que existen dos sistemas de identificación de las palabras. Uno es el lexicológico y el otro, el fonológico. El lexicológico parece utilizar un sistema de acceso al significado que implica la palabra completa, es decir incorpora procesos de asociación básicamente visuales, como sucede con el kanji (Koyama, Hansen y Stein, 2008). Por otra parte, 
y de forma alternativa, el sistema fonológico parece asociarse al deletreo de palabras que son menos utilizadas y que por tanto implican procesos asociativos, algo parecido a lo que sucede con los kanas, el silabario japonés (Vega y Soto, 2000).

No obstante, no existe absoluto consenso con relación a que la fonología sea esencial en la lectura de un sistema de escritura logográfico. Para Mori (1998), aunque una unidad morfémica está asociada con una forma fonética, el sistema morfográfico dista mucho de ser igual a un sistema fonográfico. Una razón es que no existe una relación uniforme entre grafema y fonema. Ni siquiera en el caso del chino, en donde cada carácter tiene sólo una pronunciación, deja de ser bastante vaga dicha relación, pues de hecho existen muchos más caracteres que número de sílabas usadas en chino. Algo que no pasa en un sistema fonográfico, como los kanas del japonés o el alfabeto del español. Por su parte, el japonés es fonológicamente mucho más ambiguo que el chino. No sólo no existe una relación cerrada entre grafema y sonido, sino que, además, un carácter puede presentar más de una lectura, en muchos casos hasta tres o cuatro. La correcta lectura dependerá del contexto en el cual el carácter aparece.

En virtud de estas desventajas fonológicas que presenta el sistema morfográfico, varios estudios se han concentrado en esclarecer qué estrategias siguen los lectores. Algunos de estos estudios empíricos han sugerido que las palabras escritas en kanji son procesadas de forma distinta a como sucede con los kanas (escritura fonográfica), por parte de lectores nativos del japonés (Simamura 1987; Yamada, Mitarai y Yoshida, 1991). Sasanuma (1974) concluyó a través de estudios clínicos con pacientes afásicos que, a pesar de que los sujetos no eran capaces de interpretar escritura fonográfica, sí podían reconocer el significado de las palabras escritas en kanji. De la misma forma, el estudio experimental de Osaka (1990) demostró que el significado de los kanji puede ser reconocido a través de un proceso semántico directo que no compromete el aspecto fonológico. Obana (1997) lo llama «procesamiento paralelo», proceso cognitivo que decodifica el grafema en un código semántico de forma directa. En el caso del chino, Zhou y otros (1999) demostraron que el procesamiento fonológico automático no implica necesariamente que la fonología medie la activación semántica. La razón es que la relación entre ortografía y fonología es tan inconsistente como la relación entre 
ortografía y significado. De acuerdo a estas evidencias, la ruta entre ortografía e información semántica se visualiza más fiable que la ruta entre ortografía y fonología (Matsumoto, 2013).

Si las teorías con respecto a la ruta que sigue el lector nativo de un sistema de escritura logográfico no son concluyentes, ¿Qué podría decirse de los lectores no nativos? En la sección anterior, donde se presentó la exposición del problema, se expuso de forma general algunos antecedentes sobre esta pregunta. A continuación, se aportan otros datos que contribuyen a dar un mejor marco referencial sobre el problema.

Además de los estudios citados anteriormente, resulta relevante los hallazgos de Tamaoka (1997), quien descubrió que los lectores chinos tenían menos dificultades en reconocer las palabras en japonés que sus pares angloparlantes cuando estas estaban escritas en kanji, pero que no presentaban ninguna diferencia cuando esas palabras estaban escritas en kanas (silabario japonés). La conclusión: los participantes chinos, habituados a un sistema logográfico (no exactamente el mismo que el del japonés, en todo caso) usaban de forma automática la vía visual para el reconociendo de las palabras, por el contrario de los participantes estadounidenses que al parecer se fijaban más en el aspecto fonológico de la ortografía cuando leían kanji. De la misma forma Komori (2007) comprobó que los lectores no nativos de japonés cuya lengua materna tiene una escritura alfabética tienden a depender a mayor grado de la información fonológica al momento de leer kanji.

De acuerdo con Mori (1998), la investigación en psicología experimental en adquisición del lenguaje y en lingüística ha establecido básicamente que el lector de cualquier lenguaje usa códigos fonológicos para acceder a la memoria de corto plazo que le permite acceder al significado de un texto. No obstante, se reconoce que existen diferencias estructurales entre un sistema fonográfico y morfográfico que podría provocar que lectores habituados a sus respectivos sistemas de escritura usen las mismas estrategias para tratar de interpretar códigos fonológicos en representaciones visuales. Según su estudio, los lectores con antecedentes morfográficos usan estrategias más flexibles para decodificar claves fonológicas en caracteres nuevos en comparación a como lo hacen aquellos lectores con antecedentes fonográficos. Koda (1990) llama a estos caracteres indescifrables (con respecto al sonido) para los sujetos acostumbrados a un sistema fonográfico «símbolos 
inaccesibles fonológicamente». Esto debido a que, esos caracteres no presentan ninguna pista de cómo se leen.

Como ha quedado claro hasta aquí, una revisión exhaustiva de la bibliografía disponible indica que, lejos de estar claro cuáles son los procesos cognitivos que siguen los lectores nativos de un sistema de escritura logográfico, como el utilizado en el japonés, existe aún bastante controversia con respecto a cómo el lector accede al significado y cuán importante es el aspecto fonológico en el reconocimiento de las palabras, esto último es clave para llevar a cabo una comprensión de lectura eficaz. Hasta ahora, lo que se puede concluir es que los lectores nativos establecen fuertes conexiones entre los tres aspectos involucrados en la lectura: el ortográfico, el fonológico y el semántico. Y por su parte, los estudiantes de japonés como segunda lengua aplican a la lectura las estrategias a las que están acostumbrados: en el caso de los estudiantes chinos, la evidencia muestra que el aspecto visual juega un papel preponderante, mientras que los estudiantes cuya lengua utiliza un sistema de escritura alfabético, como los hispanohablantes, utilizan distintas estrategias al momento de identificar las palabras de un sistema morfográfico, aunque prefieren buscar claves fonológicas en su lectura. Además, es necesario precisar si los sujetos adoptan estas estrategias de forma inalterable, prescindiendo de la etapa de aprendizaje en que se encuentren, o bien si a medida que van alcanzando mayor manejo del idioma sus estrategias van cambiando.

\section{Metodología}

\subsection{Participantes}

Los participantes fueron nueve estudiantes de japonés como segunda lengua. Cada uno de ellos había estudiado al momento de la aplicación de las pruebas Japonés Básico por un periodo de tres semestres y habían rendido y aprobado el Nivel 5 de la Prueba de Suficiencia en Lengua Japonesa (Nihongo Noryoku Shiken, única prueba oficial de suficiencia en lengua japonesa que va desde el Nivel 1 al 5, siendo del nivel 1 el más complejo y el nivel 5 en más elemental). Este criterio sirvió para asegurar que los participantes conocían los caracteres incluidos en las pruebas. 


\subsection{Materiales}

Se aplicaron tres diferentes pruebas más una encuesta. Las pruebas tenían la finalidad de establecer de qué forma les resultaba más rápido y preciso a los lectores reconocer el significado de los caracteres, si era sólo visualmente o bien la vía fonológica era la que permitía reconocer su sentido. La encuesta permitió determinar las creencias de los participantes sobre el sistema de escritura japonés.

La primera prueba era un test tipo Protocolo de Pensamiento en Voz Alta. Esta prueba permitió a los participantes exteriorizar lo primero que pensaban al momento de ver una palabra escrita en kanji. Básicamente, consistió en un set de letras y palabras presentadas en una pantalla por cinco segundos, en las que los participantes debían decir en voz alta lo primero que pensaban al ver el carácter: podían pronunciarla (lo que corresponde a su lectura) o bien decir su significado en español (Macaro, 2001).

La segunda prueba tenía como objetivo medir qué comprendían más rápido los participantes: información expuesta en escritura fonográfica o en escritura logográfica. El experimento consistía en una prueba de alternativas presentada en papel en la que tenían que indicar la palabra con el significado opuesto. Estaba compuesta de dos partes: la primera era un listado de palabras escritas en kanji con cuatro alternativas cada una. Entre las cuatro alternativas sólo una correspondía al significado opuesto de la palabra en cuestión. La segunda parte era un listado de palabras escritas en hiragana con sus correspondientes alternativas. Las palabras en kanji e hiragana eran distintas en cada prueba, pero relacionadas en término de tipos de palabras. Por ejemplo, la pregunta (1) era un adjetivo en cada sección, la pregunta (4) era un sustantivo relativo a persona en cada sección, la pregunta (7) era un verbo en cada sección. Se solicitó a los participantes responder la prueba lo más rápido posible con el fin de determinar qué les resultaba más rápido: reconocer una palabra escrita en hiragana (escritura fonográfica) o bien escrita en kanji. Este tipo de prueba permite evaluar la comprensión del kanji sin necesidad de que el evaluando use, en este caso, español (Kano y otros, 2001).

La tercera prueba permitió averiguar qué tipo de escritura causa una mayor fijación de significado en el lector, es decir, qué recordaban con mayor precisión los participantes: aquellas oraciones escritas 
con escritura fonográfica (hiragana) o bien texto escritos en kanji. La prueba consistió en la presentación de un set de oraciones escritas sólo en hiragana y luego otro set de oraciones paralelas, configuradas con los mismos elementos gramaticales, pero con distinto sentido, escritas en kanji. En otras palabras, las oraciones estaban construidas con la misma forma sintáctica, pero para cada parte de la oración (sujeto, objeto, circunstanciales, predicados, etc.) se utilizaron palabras distintas. Después de la lectura de cada oración, el investigador solicitaba al participante que describiera lo que había leído. Este tipo de pruebas han sido utilizadas con éxito en estudios sobre el efecto de la lectura kanji en la activación cerebral (Buchweitz y otros, 2009). Las características del japonés se constituyen en una posibilidad única para medir esto, pues como cuenta con dos sistemas de representación de la escritura hace posible constatar si la activación fonológica efectivamente juega un rol trascendental en el reconocimiento de las palabras.

Además, este experimento permitió aplicar una prueba de reconocimiento de palabras en contexto. Existen buenas razones para este tipo de prueba. Una de ellas, entiende que el reconocimiento de palabras es una parte de un proceso mucho más complejo: la comprensión de lectura. De hecho, el objetivo último de la lectura es justamente la comprensión. Las evidencias demuestran que la falta de habilidades en el reconocimiento de las palabras inciden en una deficiente comprensión de lectura (Stanovich, 2000). Por supuesto, existen otros factores que también influyen, como la sintaxis, pero estos fueron controlados durante el estudio. Además, como se ha establecido en el caso del inglés, los lectores reconocen una palabra de forma más rápida y exacta cuando esta aparece en una oración semánticamente congruente o sintácticamente correcta, o en ambos casos. Mientras que su reconocimiento resulta más complicado cuando no se dan dichas condiciones (Jordan y Tomas, 2002). Otro aspecto relevante, es el uso de pasajes que proveen contexto permite examinar las diferencias en el desarrollo de la automaticidad del reconocimiento de las palabras en una segunda lengua de forma más clara que en el formato tradicional de palabras aisladas (Chikamatsu, 2006).

Por último, la encuesta, que consistió en un cuestionario pre y post experimental, resultó ser un instrumento apropiado para determinar las creencias de los participantes con respecto a cómo ven el aprendizaje de 
kanji y, sobre todo, conocer el concepto que manejan sobre la escritura morfográfica (Mori, 1999). A la vez, permitió conocer la evaluación que hacían los propios participantes de su desempeño en las tareas realizadas.

\section{Resultados}

En primer lugar, se hará referencia a lo que reveló la encuesta pre experimento que sirvió para dar cuenta sobre las creencias de los participantes sobre el sistema de escritura kanji. Los nueve participantes, sin excepción, conciben el sistema de escritura logográfico del japonés como un sistema intrincado de «símbolos» que representan ante todo significados. Por lo tanto, están convencidos de que, al ver un carácter logográfico kanji, lo procesan como una imagen y lo decodifican directamente como una información semántica sin pasar necesariamente por su fonología.

La Tabla 1 muestra en porcentajes las respuestas de la primera prueba en la que los participantes debían indicar en voz alta la pronunciación o el significado en español de la palabra que aparecía en la pantalla.

Tabla 1. Enunciación de lectura v/s significado

\begin{tabular}{|c|c|c|c|}
\hline Participante & Lectura & Significado & Error u Omisión \\
\hline 1 & $65 \%$ & $0 \%$ & $35 \%$ \\
\hline 2 & $45 \%$ & $35 \%$ & $20 \%$ \\
\hline 3 & $75 \%$ & $10 \%$ & $15 \%$ \\
\hline 4 & $70 \%$ & $25 \%$ & $5 \%$ \\
\hline 5 & $40 \%$ & $50 \%$ & $10 \%$ \\
\hline 6 & $60 \%$ & $15 \%$ & $25 \%$ \\
\hline 7 & $65 \%$ & $0 \%$ & $35 \%$ \\
\hline 8 & $75 \%$ & $15 \%$ & $10 \%$ \\
\hline 9 & $75 \%$ & $10 \%$ & $15 \%$ \\
\hline
\end{tabular}

Como se puede ver en la Tabla 1, ocho de los nueve participantes expresaron primero mayoritariamente la pronunciación de los caracteres. Cabe destacar que del total de preguntas que no fueron respondidas o en las que erraron, sólo un 12\% correspondieron a errores, es decir, los participantes cuando no recordaban el significado, tampoco recordaban 
la lectura o viceversa. En promedio, los participantes se inclinaron a dar primero la pronunciación que el significado de las palabras en un 63,3\% de las veces y sólo el $17,7 \%$ lo hicieron de forma inversa. Una característica importante de la prueba es que el 65\% de las palabras presentadas correspondían a palabras semánticamente transparente compuestas por dos kanji, y de ellas 35\% eran palabras cuya pronunciación son una excepción a la norma, por cuanto, aunque son palabras compuestas, su lectura es japonesa (kunyomi) y no china (onyomi), pues son palabras wago, es decir, de origen japonés. Lo esperado en este caso es que los participantes se inclinaran a dar el significado, pues la lectura les resultaba claramente más compleja por tratarse de excepciones. Sin embargo, los resultados muestran que ante los estímulos, los participantes fueron proclives la mayoría de las veces a acceder al significado del kanji a través de la pronunciación del mismo. Es necesario aclarar que luego de cada ejercicio se constataba que los participantes conocieran con exactitud el significado de aquellas palabras en las que habían indicado primero su lectura (Matsumoto, 2013).

La Tabla 2 muestra el tiempo en segundos en que cada participante demoró en realizar la tarea de identificar palabras con significado opuesto entre cuatro alternativas, primero palabras escritas en kanji y luego palabras escritas en hiragana.

Tabla 2. Identificación de palabras con significado opuesto escritas en kanji y hiragana

\begin{tabular}{|c|c|c|}
\hline Participante & $\begin{array}{c}\text { Segundos para palabras } \\
\text { en } \text { kanji }\end{array}$ & $\begin{array}{c}\text { Segundos para palabras en } \\
\text { hiragana }\end{array}$ \\
\hline 1 & 21 & 24 \\
\hline 2 & 56 & 41 \\
\hline 3 & 62 & 49 \\
\hline 4 & 36 & 28 \\
\hline 5 & 47 & 34 \\
\hline 6 & 48 & 50 \\
\hline 7 & 38 & 48 \\
\hline 8 & 61 & 56 \\
\hline 9 & 71 & 64 \\
\hline
\end{tabular}


Como se puede apreciar, sólo tres de los nueve participantes demoraron menos tiempo en terminar la tarea en kanji que en hiragana. Es importante señalar que una vez terminada la tarea, se les preguntó a los participantes qué les había resultado más fácil y rápido identificar, si las palabras escritas en kanji o las escritas en hiragana. Según la opinión de los examinados, la tarea de identificar palabras en kanji resultó «mucho más fácil y rápida debido a que visualmente pueden ver de forma inmediata el significado». En contraste, en el caso de las palabras escritas en hiragana les resultaba más inconveniente porque no podían ver el significado de forma tan instantánea. A pesar de este convencimiento por parte de los participantes, los resultados de esta prueba evidencian que las palabras en caracteres fonográficos son asimiladas de forma más rápida que aquellas en caracteres logográficos. Esto podría interpretarse como una evidencia de que los kanji no necesariamente son decodificados de forma inmediata como información semántica, o por lo menos no de forma más inmediata que la escritura fonológica.

Finalmente, la prueba de reconocimiento de palabras en contexto expone datos relevantes a la hora de contrastar las estrategias de lectura de palabras aisladas y palabras que aparecen dentro de un contexto. La Tabla 3 muestra los resultados obtenidos en esta tarea.

Tabla 3. Partes de la oración omitidas en las oraciones en $\mathrm{kanji}$ v/s en hiragana

\begin{tabular}{|c|c|c|}
\hline Participante & $\begin{array}{c}\text { Partes omitidas de las } \\
\text { oraciones en hiragana }\end{array}$ & $\begin{array}{c}\text { Partes omitidas de las } \\
\text { oraciones en } \text { kanji }\end{array}$ \\
\hline 1 & 0 & 0 \\
\hline 2 & 2 & 1 \\
\hline 3 & 9 & 2 \\
\hline 4 & 5 & 0 \\
\hline 5 & 1 & 0 \\
\hline 6 & 7 & 0 \\
\hline 7 & - & - \\
\hline 8 & 2 & 0 \\
\hline 9 & 5 & 0 \\
\hline
\end{tabular}


Como se puede constatar en la Tabla 3 los participantes lograron una mayor fijación en las palabras escritas en kanji que en las escritas en hiragana. De hecho, todos los participantes, excepto uno, tuvieron problemas para recordar alguna parte de las oraciones escritas con escritura fonográfica. Uno de los examinados no logró retener el significado de una parte de la oración y otro el significado de dos. Pero los otros cinco no retuvieron cinco o más partes en el total de las oraciones leídas. Por otra parte, seis de ellos lograron retener todos las partes de las oraciones escritas en kanji. Las opiniones post experimento de los participantes, coincidieron en que les resultaba totalmente más legible un texto en kanji que en hiragana. Esto está en completa concordancia con las evidencias que muestran que en la lectura de un texto en japonés el ojo fija su atención preferentemente en la escritura logográfica (Kajii y otros, 2001). Cabe señalar, que de las tres pruebas esta fue la única en la que los resultados del ejercicio coincidieron con lo que creían los examinados.

\section{Conclusiones}

A modo de conclusión general, se puede decir que la idea preconcebida que tienen los aprendices del japonés escrito es que ellos pueden lograr comprender el significado de los caracteres pasando por alto la ruta fonológica. Están convencidos de que al leer un texto en japonés, procesan los caracteres como una imagen logográfica y los decodifican directamente como una información semántica. Cabe destacar que piensan de esta manera, a pesar de que, dentro de su formación inicial en escritura logográfica, se enfatiza la exposición de los planteamientos que ven la escritura kanji, esencialmente, como una forma más de escritura fonológica (Maeth, 1987; Unger, 1990) o como la denomina Matsunaga (1996) morfofónica. Los participantes saben que, estadísticamente hablando, cerca del $70 \%$ de los caracteres utilizados de forma oficial en los textos en japonés corresponden a kanji «fonéticos», es decir que entregan alguna pista sobre cómo se leen. No obstante a eso, su concepción de que kanji es un carácter que decodifican semánticamente de forma inmediata es un concepto fuertemente arraigado. Una explicación de esto podría ser que buena parte de los primeros kanji que ellos aprenden son caracteres de tipo pictográficos e ideográficos (Matsunaga, 1996). Por lo tanto, ven los kanji de la manera en cómo aprendieron los primeros: relacionando la forma de la letra con una 
imagen tomada del mundo real. Además, es probable que en esta etapa de su aprendizaje, el uso reiterado de estrategias como la mnemotecnia resulte en que no logren disociar las caracterizaciones que se hacen sobre la formación de los kanji y su uso real en la escritura japonesa. Como sea, la creencia compartida entre los participantes, y probablemente entre los estudiantes principiantes de japonés en general, es que no es del todo esencial recordar la pronunciación de un kanji, pues pueden procesar los caracteres como una imagen logográfica y decodificarlos directamente como una información semántica.

Por otra parte, los resultados conseguidos a través de las pruebas aplicadas en este estudio, sugieren que en el proceso de reconocer el significado de una palabra kanji la fonología es efectivamente un componente de la activación semántica. Si bien se puede presentar casos en que el lector recuerde el significado de la palabra, pero no su pronunciación, por lo general el lector es capaz de reconocer lectura y significado de forma simultánea. No obstante, dentro de un contexto, las palabras escritas en kanji efectivamente son retenidas de forma más permanente, esto seguramente por las características propias de un sistema logográfico que es intrínsecamente visual. Esto concuerda con Jordan y Tomas (2002) quienes sostienen que los lectores reconocen una palabra de forma más rápida y exacta cuando esta aparece en una oración semánticamente congruente o sintácticamente correcta, o en ambos casos.

Las pruebas magnetoencefalográficas y las imágenes por resonancia magnética funcional han demostrado que los perfiles de activación cerebral son distintos cuando el lector lee kanji versus otros tipos de escritura fonográfica (Buchweitz y otros, 2009; Valaki y otros, 2003). No obstante, aún no es posible determinar de forma categórica cómo acceden al significado los lectores no nativos de un sistema logográfico y si pueden prescindir de la ruta fonológica. Este estudio sugiere que los estudiantes principiantes de japonés tienden a acceder al significado por la vía fonológica y visual simultáneamente, a pesar de su firme convicción de que al leer procesan los caracteres kanji como una imagen logográfica y los decodifican directamente como una información semántica. Por otro lado, cuando el reconocimiento de las palabras se lleva a cabo dentro de un contexto la ruta entre ortografía e información semántica se visualiza más fiable que la ruta entre ortografía y fonología. 


\section{Referencias bibliográficas}

Akamatsu, N. (2002). A similarity in word-recognition procedures among second language readers with different first language backgrounds. Applied Psycholinguistics, 23, 117-133.

Baron, J. (1973). Phonemic stage not necessary for reading. Quarterly Journal of Experimental Psychology, 25, 241-246.

Buchweitz, A. (2009) Japanese and English sentence reading comprehension and writing systems: An fMRI study of first and second language effects on brain activation. Biling, 12, 141-151.

Carrel, P. (1989). Metacognitive awareness and second language reading. Modern Language Journal, 73, 121-134.

Chikamatsu, N. (1996). The effects of L1 orthography on L2 word recognition: A study of American and Chinese learners of Japanese. Studies in Second Language Acquisition, 18, 403-432.

Chikamatsu, N. (2006). Developmental word recognition: A study of L1 English readers of L2 Japanese. The Modern Language Journal, 90, 67-85.

Coltheart, M. (1978). Lexical access in simple reading tasks. En G. Underwood (Ed.), Strategies of information processing. San Diego, CA: Academic Press.

DeFrancis, J. (1989). Visible speech. The diverse oneness of writing systems. Honolulu: University of Hawai'i Press.

Durgunoglu, A. Y., \& Oney, B. (1999). A cross-linguistic comparison of phonological awareness andword recognition. Reading and Writing: An Interdisciplinary Journal, 11, 281-299.

Frost, R. (1994). Prelexical and postlexical strategies in reading: Evidence from a deep and a shallow orthography. Journal of Experimental Psychology: Learning, Memory, and Cognition, 29, 116-129.

$\mathrm{Gu}$, Y. y Johnson, K. (1996). Vocabulary learning strategies and language learning outcomes. Language Learning, 46, 643-679.

Jordan, T. R., \& Thomas, S. M. (2002). In search of perceptual influences of sentence context on word recognition. Journal of Experimental Psychology: Learning, Memory, and Cognition, 28, 34-45. 
Kajii, N. y otros (2001). Eye movement control in reading anspaced text: The case of the Japanese script. Vision Research, 41, 2503-2510.

Kano, C. y otros. (1993) Intermediate KanjiBook. Vol. 1. Bonjinsha, Tokyo.

Kess, J.F., \& Miyamoto, T. (1999). The Japanese mental lexicon: Psycholinguistic studies of kana and kanjiprocessing. Philadelphia/ Amsterdam: John Benjamins.

Koda, K. (1990). The use of L1 reading strategies in L2 reading. Studies in Second Language Acquisition, 12, 393-410.

. (1996). L2 recognition research: A critical review. The Modern Language Journal, 80, 450-460.

. (1999). Development of L2 intraword orthographic sensitivity and decoding skills. The Modern Language Journal, 83, 51-64.

_.. (2000). Cross-linguistic variations in L2 morphological awareness. Applied Psycholinguistics, 21, 297-320.

Komori, S. (2007). Eigo wo bogoto suru chuujoukyuu nihongo gakushusha no kanjigoi no ninchi nituite: otonoeikyo (Kanji recognition of intermediate-high learners of Japanese whose first language is English: Sound effect on word recognition). En Proceedings of Nihongo kyoiku gakkai shunki taikai (The Conference of the Society for Teaching Japanese as a Foreign Language, Tokio) (pp. 119-124). Tokio.

Koyama, M. S., Hansen, P. C. \& Stein, J. F. (2008). Logographic Kanji versus Phonographic Kana in Literacy Acquisition: How Important Are Visual and Phonological Skills? Annals of the New York Academy of Sciences, 1145, 41-55.

Macaro, E. (2001). Learning strategies in foreign and second language classrooms. China: Continuum International Publishing Group.

Matsumoto, K. (2013). Kanji Recognition by Second Language Learners: Exploring Effects of First Language Writing Systems and Second Language Exposure. The Modern LanguageJournal, 97, 161-177.

Maeth, R. (1987). Sobre «La escritura del este de Asia». Estudios de Asia y África, Vol. 22(1), 62-69. 
Matsunaga, S. (1996). The Linguistic Nature of Kanji Reexamined: Do Kanji Represent Only Meanings? The Journal of the Association of Teachers of Japanese, 30, 1-22.

Mori, Y. (1998). Effect of First Language and Phonological Accessibility on Kanji Recognition. The Modern Language Journal, 82, 69-82. (1999). Beliefs about Language Learning and Their relationship to the Ability to Integrate Information from Word Parts and Context in Interpreting Novel Kanji Words.The Modern Language Journal, 83(4), 534-547.

Morita, A., \&Tamaoka, K. (2002). Phonological involvement in the processing of Japanese at the lexical and sentence levels. Reading and Writing: An Interdisciplinary Journal, 15, 633-651.

Obana, Y. (1997). Vertical or Horizontal? Reading Directions in Japanese. Bulleting of the School of Oriental and African Studies, University of London, 60, 86-94.

Osaka, N. (1990). Spread of visual attention during fixation while reading Japanese text. En Groner, R., G. d'Ydewalle and R. Parham (Ed.), From eye to mind: information acquisition in perception, search and reading. Michigan: North-Holland.

Perfetti, C.A., Zhang, S., \& Berent, I. (1992). Reading in English and Chinese: Evidence for a «universal» phonological principle. En R. Frost \& L. Katz (Eds.), Orthography, phonology, morphology, and meaning (pp. 227-248). Amsterdam: Elsevier.

Sasanuma, S. (1974). Kana and kanji processing in Japanese aphasics. Brain and Language, 2, 369-384.

Sasanuma, S., \& Fujimura, O. (1971). Selective impairment of phonetic and nonphonetic transcription of words in Japanese aphasic patients: kana vs. kanji in visual recognition and writing. Cortex, 7, 1-18.

Simamura, A. P. (1987). Word comprehension and naming: An analysis of English and Japanese orthographies. American Journal of Psychology, 100, 15-40.

Stanovich, K. E. (2000). Progress in understanding reading: Scientific foundations and new frontiers. New York: The Guilford Press. 
Tamaoka, K. (1997). The processing strategy of words presented in kanji and kana by Chinese and English speakers learning Japanese. Studies in Language and Literature, 17, 65-77.

Tokunaga, H., Nishikawa, T., Ikejiri, Y., Nakagawa, Y., Yasuno, F., Hashikawa, K., Nisimura, T., Sugita, Y., \& Takeda, M. (1999). Differential neural substrates for Kanji and Kana writing: a PET study. NeuroReport, 10, 3315-3319.

Unger, J. M. (1990). The Very Idea. The Notion of Ideogram in China and Japan. Monumenta Nipponica, 45, 391-411.

Valaki, C. y otros. (2003). Do different writing systems involve distinct profiles of brain activation? A magnetoencephalography study. Journal of Neurolinguistics, 16, 429-438.

Vanness, S. (2005). Difficult Characters: Interdisciplinary Studies of Chinese and Japanese Writing by Mary S. Erbaugh. The Modern LanguageJournal, 89, 147-148.

Vega, R., \& Soto, E. (2000). Sustratos neutrales en la escritura kana y kanji. Elementos, 37, 13.

Wydell, T.N., Patterson, K.E., \& Humphreys, G. W. (1993). Phonologically mediated access to meaning for kanji: Is a rows still rose in Japanese kanji? Journal of Experimental Psychology: Learning, Memory, and Cognition, 19, 491-514.

Yamada, J., Mitarai, Y., \&Yoshida, T. (1991). Kanji words are easier to identify than Katakana words. Psychological Research, 53, 136-141.

Zhou, X., Shu, H., Bi, Y., \& Shi, D. (1999). Is there phonologically mediated access to lexical semantic in reading Chinese? En J. Wang, A.W. Inhoff, \& H.C. Chen (Eds.), Reading Chinese script: A cognitive analysis. Mahwah, NJ: Lawrence Erlbaum. 
